\title{
The cytotoxic effect of titanium particles phagocytosed by osteoblasts
}

\author{
Dominique P. Pioletti, ${ }^{1}$ Hiroshi Takei, ${ }^{2}$ Soon Y. Kwon, ${ }^{2}$ Douglas Wood,${ }^{1}$ K-L. Paul Sung ${ }^{1,2,3}$ \\ ${ }^{1}$ Department of Bioengineering, University of California, San Diego, 9500 Gilman Drive, La Jolla, California $92093-412$ \\ ${ }^{2}$ Department of Orthopaedics, University of California, San Diego, 9500 Gilman Drive, La Jolla, California 92093-412 \\ ${ }^{3}$ Whitaker Institute for Biomedical Engineering, University of California, San Diego, 9500 Gilman Drive, \\ La Jolla, California 92093-0412
}

Received 8 June 1998; accepted 4 February 1999

\begin{abstract}
The cytotoxic effect of different concentrations of titanium particles on osteoblasts was studied in vitro. It was found that the viability of the osteoblasts was inversely proportional to the particle concentration. Phagocytosis of particles by the osteoblasts was evident and was demonstrated to be responsible for cell necrosis. Moreover, during and after phagocytosis, the osteoblasts released products that were cytotoxic for other osteoblasts, as established with a conditioned medium assay. The titanium particles thus had both a direct and an indirect effect on osteoblast viability. It
\end{abstract}

also was observed that the titanium particles induced a process of programmed cell death (apoptosis) when co-cultured with osteoblasts. The results of this study suggest that not only is the amount of wear debris generated important, but the local accumulation of the debris also may have a significant impact on bone cell function. (C) 1999 John Wiley \& Sons, Inc. J Biomed Mater Res, 46, 399-407, 1999.

Key words: osteoblast; viability; titanium particles; phagocytosis; apoptosis

\section{INTRODUCTION}

The particles generated during the normal or abnormal wear processes of an orthopedic implant have been recognized as one of the major factors responsible for aseptic implant loosening. ${ }^{1,2}$ Numerous studies have been performed to elucidate the role of particles in the biological reaction surrounding total joint implants. Particulate debris has been shown to promote fibroblast proliferation in the synovial membrane and thus are an important factor in the creation of the fibrous membrane surrounding the prosthesis. ${ }^{3}$ Moreover, the phagocytosis of the particles by different cells has been demonstrated to be indirectly responsible for peri-implant osteolysis. ${ }^{4}$ These biological reactions to particles can act in combination to weaken the mechanical anchorage of the implant and, finally, can result in its loosening, which generally requires a more complicated revision procedure.

Correspondence to: K.-L. P. Sung; e-mail: klpsung@ bioeng.ucsd.edu

Contract grant sponsor: Swiss National Science Foundation

Contract grant sponsor: OREF Bristol-Meyers; Contract grant number: 59212A

(c) 1999 John Wiley \& Sons, Inc.

CCC 0021-9304/99/030399-09
Normal bone function is assured when an equilibrium process between bone formation and bone resorption is reached. Although some mechanical phenomena, such as stress shielding, are implicated in peri-implant osteolysis, ${ }^{5}$ particles seem to be a major factor involved in the loss of balance between bone formation and bone resorption. In view of the bone remodeling process, three situations can arise; (1) Bone resorption is overstimulated by excessive inflammatory reaction to particles; (2) bone formation is inhibited by interaction with the wear particles; or (3) these two reactions act in combination. Until recently, only the first possibility has been tested either directly (with osteoclastic cells in contact with particles ${ }^{6}$ ) or indirectly (by the quantification of potent osteolytic factors produced by macrophages, ${ }^{7-9}$ lymphocytes, ${ }^{10}$ monocytes, ${ }^{11,12}$ or giant cells ${ }^{4}$ in contact with particles). To describe the effects of particles on the osteolysis problem, the co-culture of macrophage/ osteoblast in contact with titanium or polymethylmethacrylate particles has been used. ${ }^{13,14}$ The corresponding production of cytokines and mediators and their effects on bone resorption were quantified. High concentrations of titanium particles were used (up to $10 \mathrm{mg} / \mathrm{mL}$ ). However no direct osteoblastparticle contact was allowed in these studies as only the supernatant from the macrophage/particle culture 
was used for the osteoblast cultures. In vivo studies have been performed to elucidate the response of bone to high-density polyethylene or titanium particles. ${ }^{15,16}$ Depending on the technique used for these in vivo studies, a dose-response relationship for the particles either was noted ${ }^{15}$ or was absent. ${ }^{16}$ General useful information can be obtained from in vivo studies; it is, however, difficult from these studies to describe specifically the reaction of the osteoblasts only to particles.

The direct effect of particles on bone formation, that is, on osteoblastic cells, thus has not been clearly defined. If the viability of osteoblasts is adversely influenced by wear debris, the rate of bone formation will decrease. The process of bone loss around the implant then would be more severe as a combination of decreased bone formation and increased bone resorption would act in tandem when particles are present.

At particle concentrations ranging from 0.01 to 1.0 $\mathrm{mg} / \mathrm{mL}$, no cytotoxic effects on osteoblasts were found for chromium and cobalt-chromium although cytotoxicity has been induced by cobalt. ${ }^{17}$ Using osteoblasts isolated from the tissue surrounding a failed hip or a failed knee prosthesis, it has been shown that polyethylene particles decreased cell proliferation. ${ }^{18}$ The viability of osteoblasts has been shown to be independent of titanium particles tested at various concentrations ranging from 0.01 to $100 \mathrm{ng} / \mathrm{mL}^{19}$ Titanium particles at a concentration of approximately 4.0 $\mathrm{mg} / \mathrm{mL}$ also have been shown not to have any effect on the viability and the proliferation of osteoblasts, but they have been demonstrated to suppress the expression of the genes that code for collagen. ${ }^{20}$

It has been found that the number of titanium particles retrieved from tissue surrounding a loose titanium implant was much higher than the number of corresponding particles released from chrome-cobalt or stainless steel corresponding implants. ${ }^{21}$ In the worst cases, the titanium particles could represent up to $25 \mathrm{mg} / \mathrm{g}$ of the dry retrieved tissue. The high particle number observed in bone corresponded to an accumulation process of particles over time. ${ }^{22}$ The local concentration of particles then can be very high and, in that case, it is not well simulated by the relatively low concentration used in previous published studies. Titanium alloy is being used more and more in different types of implants, so it is important to study the direct reaction of osteoblasts to titanium particles at concentrations corresponding to those that have been retrieved in bone.

In the present study, the direct and indirect effects of different titanium particle concentrations on the viability of osteoblasts has been quantified. In particular, the hypothesis that the viability of osteoblasts is adversely affected by titanium particles was tested. In this case, the type of death for the osteoblasts, that is, necrosis or apoptosis, will be determined.

\section{MATERIALS AND METHODS}

\section{Cell cultures}

Neonatal calvarial rat osteoblasts were cultured at $37^{\circ} \mathrm{C}$ and $5 \% \mathrm{CO}_{2}$ in monolayer in Dulbecco's Modified Eagle Medium (DMEM, Irvine Scientific, Santa Ana, California) containing 10\% fetal bovine serum (Irvine Scientific, Santa Ana, California). These cells were characterized as being of osteoblastic phenotype by testing for the formation of calcium phosphate salts, which is an indication of osteoblast mineralization, and the presence of alkaline phosphatase. ${ }^{23}$ Furthermore, the cells displayed the elongated polygonal morphology typical of osteoblasts in culture. Osteoblasts of passages 3-6 were used for the experiments. Cells were plated in 24-well plates at a concentration of $1 \times 10^{5}$ cells/ well.

\section{Titanium particles}

Commercially pure titanium particles of -325-mesh nominal diameter were purchased (Aldrich, Milwaukee, Wisconsin). A semi-automatic image processing method using $\mathrm{NIH}$-Image allowed verification that $80 \%$ of the particle diameters were less than $5 \mu \mathrm{m}$ (mean value \pm SD: $3.1 \pm 3.6$ $\mu \mathrm{m})$.

The particles, autoclaved at $135^{\circ} \mathrm{C}$ for $15 \mathrm{~min}$, were mixed with the culture medium under sterile conditions. Based on a particle weight to medium volume ratio, different concentrations of titanium particles were prepared. Concentrations of $0.05,0.15,0.5,0.75$, and $1 \%$ of particles corresponded to $0.5,1.5,5,7.5$, and $10 \mathrm{mg}$ of titanium particles per $\mathrm{mL}$ of medium. Based on the size distribution of the titanium particles, $1 \mathrm{~mL}$ of the concentration of $0.05,0.15,0.5,0.75$, and $1 \%$ contained approximately $0.85 \times 10^{6}, 2.54 \times 10^{6}, 8.48 \times 10^{6}$, $12.72 \times 10^{6}$, and $16.97 \times 10^{6}$ particles, respectively.

The titanium solutions were ultrasonicated for $30 \mathrm{~min}$ in sealed sterile containers before being added to the cell culture.

\section{Viability of osteoblasts}

The supernatant and the cells were collected by trypsinization and simultaneously were double stained for $5 \mathrm{~min}$ at room temperature using a fluorescein diacetate (FDA) and a propidium iodide (PI) stain (Sigma, St. Louis, Missouri). ${ }^{24}$ The stained cells were examined with an inverted fluorescent microscope (Nikon, Tokyo, Japan) equipped with epiillumination (blue filter of $495 \mathrm{~nm}$ and green filter of $546 \mathrm{~nm}$ allowed visualization of the green and red fluorescing cells, respectively). The solution of stained cells was vortexed before counting, leading to a homogeneous distribution of green and red cells in each sample. A minimum of two hundred viable(green)/nonviable(red) cells was counted for each measurement. Viability was defined as the ratio of vi- 
able cells to the total number of cells (number of green cells / number of green + number of red cells).

\section{Cell-death characterization}

In order to determine if the cells in contact with titanium particles died by necrosis or by apoptosis, a method of terminal deoxynucleotidyl transferase (TdT)-mediated dUTPbiotin nick-end labeling (TUNEL) was performed. ${ }^{25}$ Briefly, the cells were collected by trypsinization, fixed in formaldehyde, and permeabelized. Then the DNA strand breaks, specific to apoptotic cells, were labeled with biotin dUTP (Molecular Probes, Eugene, Oregon) and the reaction was catalyzed by exogenous TdT (Boehringer Mannheim, Indianapolis, Indiana). The cells then were visualized under the blue light $(488 \mathrm{~nm})$ of an epi-fluorescence microscope. The apoptotic cells fluoresced green. In order to obtain quantitative apoptosis data, the green cells were counted first and all the cells then were counterstained with PI. This latter stain allowed visualization of all the cells (since they previously had been permeabilized). At least two hundred cells were counted for each measurement. Three different culture conditions were tested with the TUNEL assay. The osteoblasts were incubated for $72 \mathrm{~h}$ with normal medium, defining the control group, with $1.0 \%$ titanium particle concentration, and with serum-free medium defining the positive apoptotic cell control group. The $1 \%$ concentration of particles was used since it was assumed that the highest concentration would be the most cytotoxic for the osteoblasts. As anticipated, it was shown that the serum-free medium induced osteoblast apoptosis. ${ }^{26}$ The apoptotic rate was defined as the ratio of the number of green cells to the number of red cells.

\section{Direct effect of titanium particles on osteoblast viability}

\section{Particle concentration effect}

After the osteoblasts were incubated for $4 \mathrm{~h}$, the medium was removed an $1 \mathrm{~mL}$ of new medium (in control wells) and $1 \mathrm{~mL}$ of various titanium particle concentrations in medium $(0.05,0.15,0.5,0.75$, and $1.0 \%)$ were added. The cells were incubated for $72 \mathrm{~h}$ and their viability then was measured.

\section{Time effect}

A kinetic study was performed by checking the viability of osteoblasts at 3, 6, 12, 24, 48, and $72 \mathrm{~h}$ of incubation after the titanium particles (control, $0.05,0.15,0.5$ and $1.0 \%$ ) were added.

\section{Phagocytosis assessment}

Inverted or epi-fluorescence microscopy did not allow us to determine whether the titanium particles were inside the osteoblasts (i.e., whether or not the particles had been phagocytosed) or only attached to the cell surface. The micropipette technique ${ }^{27}$ then was used to clarify this point. After the osteoblasts and a $0.15 \%$ concentration of Ti particles were seeded for $15 \mathrm{~min}$, the cells were detached with a micropipette and aspirated inside the tip of the pipette. If the particles were attached to the cell surface, the aspiration process should separate the particle from the cell as the small diameter of the pipette (5 to $6 \mu \mathrm{m}$ ) would not leave enough room between the cell surface and the pipette for the particles to be aspirated. If the particles were phagocytosed, aspiration of particles and the cell simultaneously should be possible.

The number of particles found inside the cytoplasm of the osteoblasts was counted for the 0.05 and $0.15 \%$ titanium concentration. Only these two low concentrations allowed a clear view of the osteoblast cytoplasm. The intake rate of the particles was defined as the percentage of osteoblasts with ingested titanium particles relative to the total number of osteoblasts. The particle intake rate was reported at 3, 6, 12, 24,48 , and $72 \mathrm{~h}$ after adding the particles. The experiment was performed four times, with a minimum of 200 cells counted at each time step.

\section{Phagocytosis effect}

In order to evaluate the effect of particle-induced phagocytosis on the osteoblast viability, the cells were treated with cytochalasin-D. This compound is known to inhibit phagocytosis by affecting the actin filament assembly of the cytoskeleton. ${ }^{28}$ After the cells were seeded and incubated for $4 \mathrm{~h}$, the medium was removed from each well and $0.5 \mathrm{~mL}$ of new medium (in control wells) and $0.5 \mathrm{~mL}$ of cytochalasin-D solutions $(1.25$ or $5 \mu M)$ were added into the wells. The cells were incubated for $1 \mathrm{~h}$ and $0.5 \mathrm{~mL}$ of new medium (in control wells) and $0.5 \mathrm{~mL}$ of titanium particle solution (final concentration $1 \%$ ) were added. The cells were incubated for $72 \mathrm{~h}$ and their viability then measured.

\section{Indirect effect of titanium particles on osteoblast viability}

\section{Particle concentration effect}

After the osteoblasts with and without titanium particles had been incubated for $72 \mathrm{~h}$, in parallel to the viability measurement, the supernatant of each well individually was collected and centrifuged for $5 \mathrm{~min}$ at $500 \mathrm{~g}$ (to avoid the contamination of the supernatant by particles). A second group of osteoblasts was incubated for $4 \mathrm{~h}$, and the medium was removed from each well. The supernatant collected from the first osteoblast group then was used as the culture medium. The second cell group, cultured with the conditioned medium, was incubated for $72 \mathrm{~h}$ and its viability then measured.

In order to verify that no cytotoxicity was induced solely from the contact of titanium particles with the medium or from the supernatant, an identical procedure was repeated 
with the titanium particle solution only. The $1.0 \%$ titanium particle solution (without cells) was incubated for $72 \mathrm{~h}$, aspirated, and then added (without particles) to osteoblasts seeded in another 24-well plate. The osteoblasts cultured with this particle-conditioned medium were incubated for another $72 \mathrm{~h}$ and their viability measured.

\section{Phagocytosis effect}

To study the indirect effect of phagocytosis, it was important to avoid the transfer of cytochalasin-D in the supernatant. The following procedure was used. After the cells were incubated for $4 \mathrm{~h}$, the medium was removed from each well and $1 \mathrm{~mL}$ of new medium (in control wells) and $1 \mathrm{~mL}$ of cytochalasin-D solution $(1.25$ or $5.0 \mu \mathrm{M})$ were added to the wells. The cells were incubated for $1 \mathrm{~h}$ and the medium was removed. Each well was washed twice with medium, and then $1 \mathrm{~mL}$ of new medium (in control wells) and $1 \mathrm{~mL}$ of $1.0 \%$ titanium particle concentration were added. The cells were incubated for $72 \mathrm{~h}$ and their supernatant then transferred to a second osteoblast culture in the same way as described above for measurement of cytoxocity. The second cell group cultured with the conditioned medium was incubated for $72 \mathrm{~h}$ and the cell viability then measured.

\section{Statistical analysis}

The kinetic studies were performed three times while all the other experiments were performed four times in quadruplicates, yielding essentially identical results. A one-way ANOVA test was used to analyze the mean variance of viability. If statistically significant differences were found, a least significant difference (Fisher $t$ test) was performed to determine which means were different from which other means. ${ }^{29}$ A 95\% confidence level was selected to define significance for all statistical tests.

\section{RESULTS}

The osteoblasts in contact with titanium particles began a process of phagocytosis. After $72 \mathrm{~h}$ of coculturing osteoblasts with titanium particles, several particles were found inside the osteoblasts (Fig. 1). The morphology of the osteoblasts changed according to the size and number of particles ingested. The osteoblasts were less elongated after they phagocytosed the particles. It also was observed that the osteoblasts phagocytosed most of the particles in the first $24 \mathrm{~h}$, as shown by the particle intake rate (Fig. 2).

The phagocytosis of particles was confirmed by micropipette manipulation, which clearly showed that the particles were inside the cell after their aspiration in the pipette (Fig. 3).

The viability of the osteoblasts was influenced by direct contact with the titanium particles in a concen-

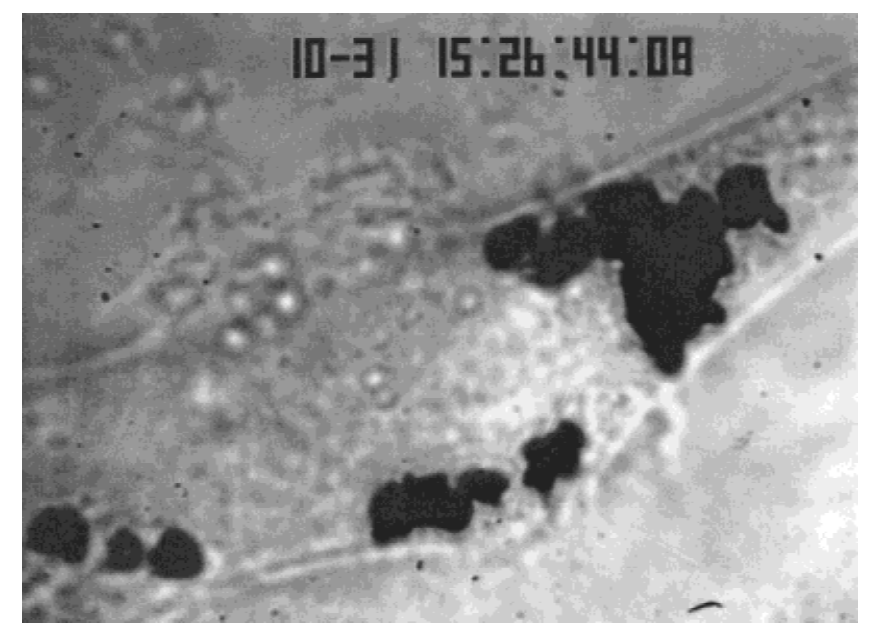

Figure 1. View (original magnification $\times 4000$ ) of an osteoblast with titanium particles in its cytoplasm after $72 \mathrm{~h}$ of co-incubation with an $0.15 \%$ concentration of $\mathrm{Ti}$ particles.

tration-dependent manner (Fig. 4). In the range of particle concentrations used, a linear inverse relationship between the concentration and the viability was found (coefficient of linear regression, $\mathrm{R}=0.98$ ). At low particle concentration $(0.05 \%)$, no statistical differences were found between the mean viability of control and the particle-osteoblast co-cultures, but at higher concentrations $(0.15,0.5,0.75$, and $1.0 \%)$ statistical differences were observed.

A time dependence of osteoblast viability was observed (Fig. 5). Especially for the 1\% particle concentration the viability decreased rapidly. At all particle concentrations, osteoblast viability decreased until a plateau was reached after $24 \mathrm{~h}$.

The viability of cytochalasin-D-treated particleosteoblast co-cultures statistically increased when compared to the viability of particle-osteoblast cocultures not treated with cytochalasin-D (Fig. 6). This

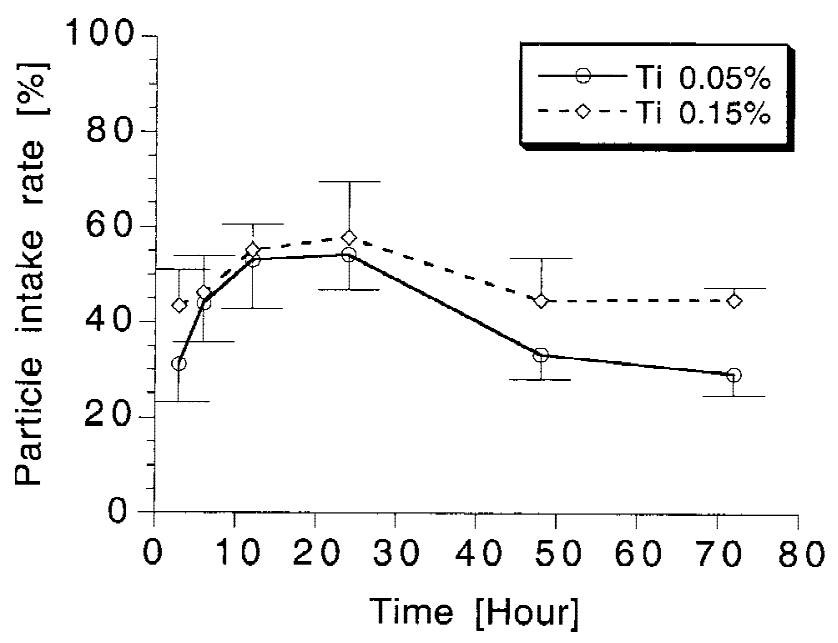

Figure 2. Mean values and standard errors of particle intake rate for the 0.05 and $0.15 \% \mathrm{Ti}$ particle concentrations at $3,6,12,24,48$, and $72 \mathrm{~h}$ after adding the particles $(n=4)$. 

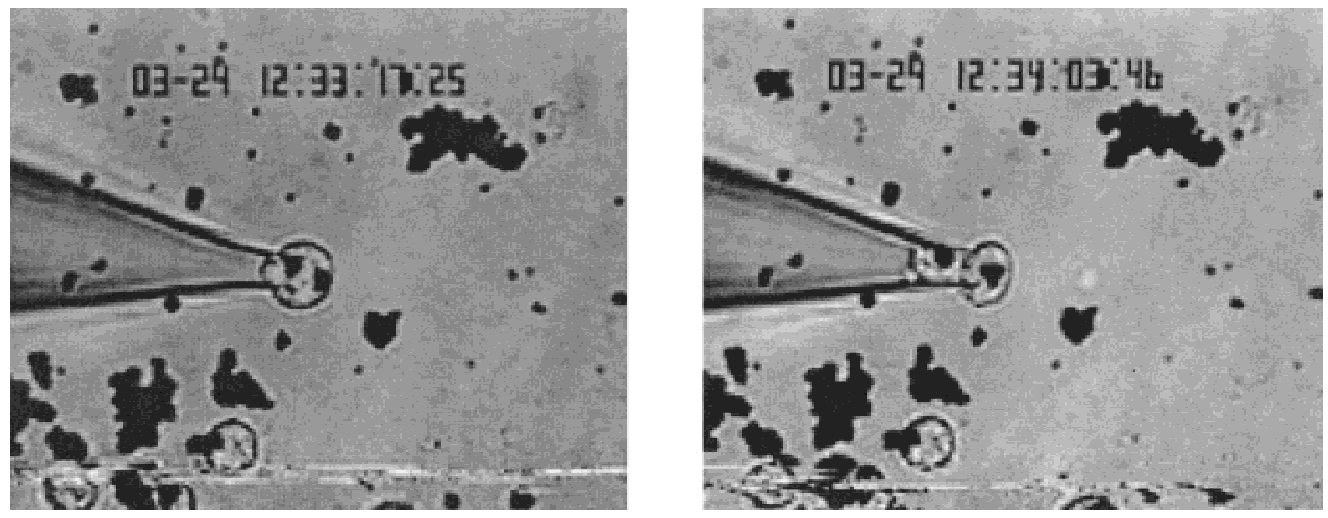

Figure 3. Micropipette technique used to assess that the Ti particles have been phagocytosed by osteoblasts. After the osteoblasts and the Ti particles at a concentration of $0.15 \%$ were seeded for $15 \mathrm{~min}$, the aspiration of the osteoblast inside the pipette highlighted that the particles were still inside the cell at the end of the process.

result indicates that the phagocytosis of titanium particles by osteoblasts is a factor in the decrease of their viability. However, statistical differences still were observed between the viability of the control and the particle-osteoblast co-cultures with cytochalasin-D while no statistical differences were found between the effects of the two different concentrations of cytochalasin-D.

The number of apoptotic cells in contact with titanium particles was shown to be significantly increased compared to the control (Fig. 7). Used as positive apoptosis controls, the osteoblasts cultured without serum also showed an increased in the proportion of apoptotic cells compared to the control.

Prior to testing the indirect effect of titanium particles, it was important to verify that no cytotoxicity was induced by the interaction of particles with medium or by the supernatant. No statistical differences were found between the viability of osteoblasts cultured with the control-conditioned medium and with the medium conditioned from incubation with titanium particles (data not shown).

Incubation of osteoblasts with particle-conditioned

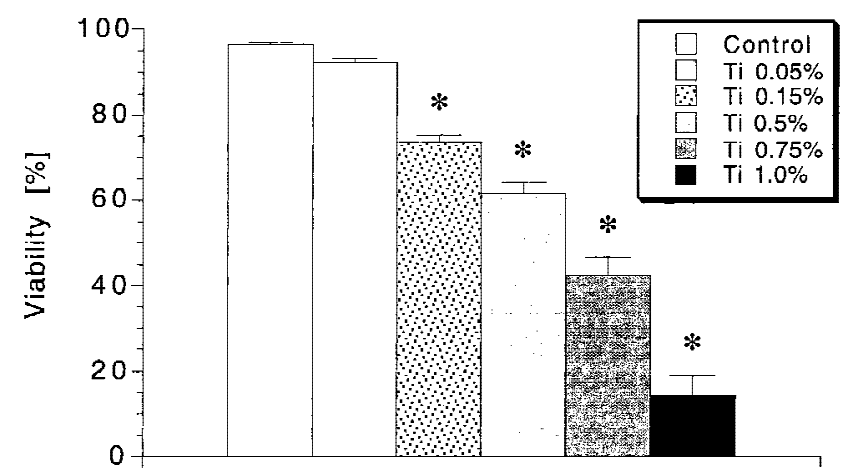

Figure 4. Mean values and standard errors of osteoblast viability after $72 \mathrm{~h}$ of co-incubation with different Ti particle concentrations (*: statistical differences with respect to control, $n=16$ and $p<0.0005)$. medium indicated that the titanium particles also had an indirect effect on osteoblast viability (Fig. 8). This effect was dependent on the initial titanium particle concentration. A threshold value of initial titanium particles between 0.75 and $1 \%$ was shown to statistically decrease the viability of the osteoblasts cultured with the conditioned medium.

The viability of osteoblasts cultured with the conditioned medium from the particle-osteoblast co-culture treated with cytochalasin-D statistically increased when compared to the corresponding results with no cytochalasin-D treatment (Fig. 9). However, statistical differences were observed between the viability of osteoblasts cultured with the control-conditioned medium and with the conditioned medium from particle-osteoblast co-cultures treated with cytochalasin-D. No statistical differences were found between the effects of the two different concentrations of cytochalasin-D.

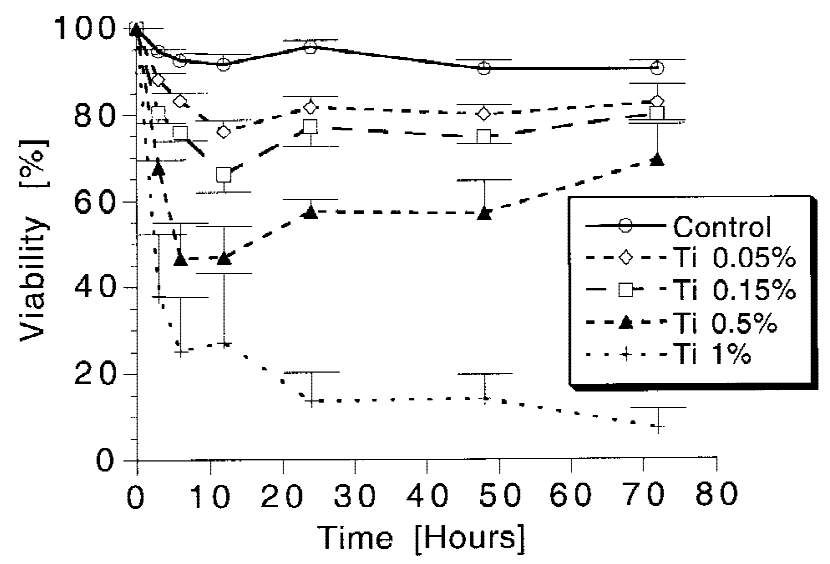

Figure 5. Mean values and standard errors of osteoblast viability as a function of time after the addition of Ti particles. Within $12 \mathrm{~h}$ after adding the particles, the viability of the osteoblasts decreased $(n=3)$. 


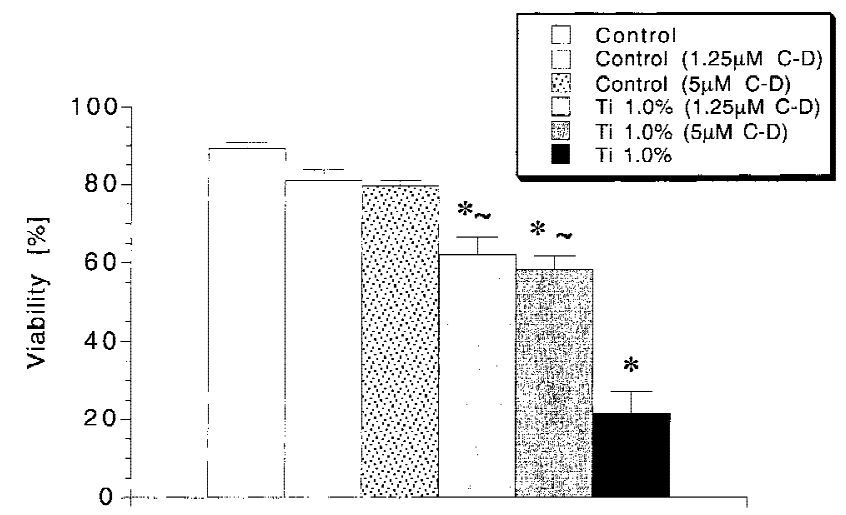

Figure 6. Mean values and standard errors of osteoblast viability when treated with cytochalasin-D after $72 \mathrm{~h}$ of incubation (*: statistical differences with respect to control, $n=$ 16 and $p<0.0005 ; \sim$ : statistical differences with respect to $1 \%$ Ti without cytochalasin-D, $n=16$ and $p<0.0005)$.

\section{DISCUSSION}

In this study, the direct and indirect cytotoxic effects of titanium particles on osteoblasts were confirmed. It also was shown that osteoblasts initiate a process of programmed cell death (apoptosis) when particles are present in culture.

Our results relative to the direct effect of exposure correlated with previous published studies, where it was observed that low concentrations of titanium particles have no effect on osteoblast viability. ${ }^{19,20}$ However, we found that the viability of osteoblasts was affected when the particle concentration increased. It thus can be speculated that osteoblasts can ingest only a restricted number of particles without being damaged. This kind of critical concentration value promoting osteolysis has been identified for polyethylene particles. ${ }^{30}$ For their direct effect on osteoblast viability, the high concentration of particles may be a more important factor than the composition of the particles. We repeated the study with titanium oxide $\left(\mathrm{TiO}_{2}\right)$ and silica $\left(\mathrm{SiO}_{2}\right)$ particles of identical size distribution as $\mathrm{Ti}$

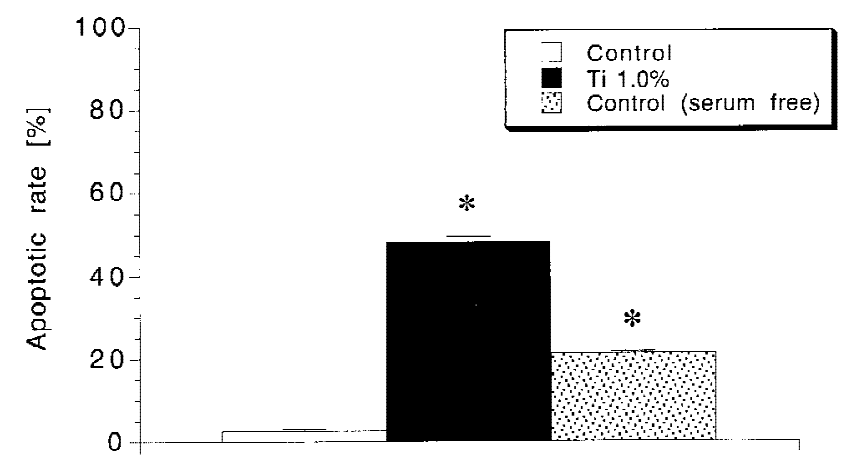

Figure 7. Mean values and standard errors of osteoblast apoptotic rate in three situations, determined with the TUNEL assay $(*$ : statistical differences with respect to control, $n=16$ and $p<0.0005)$.

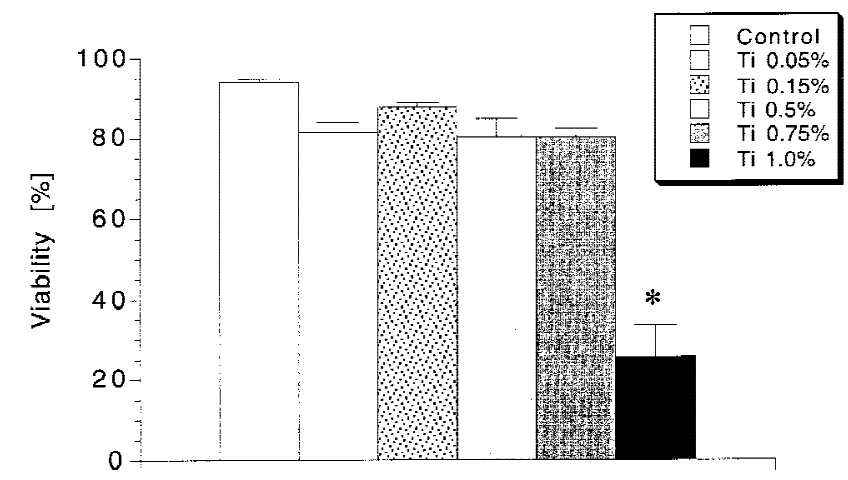

Figure 8. Mean values and standard errors of osteoblast viability when cultured with conditioned medium issued from co-cultured osteoblasts and Ti particles (*: statistical differences with respect to control, $n=16$ and $p<0.025)$.

particles, using the highest concentration $(10 \mathrm{mg} / \mathrm{mL})$. The results relative to their direct effect on osteoblast viability were similar to those of the Ti particles (data not shown). However no indirect effect on osteoblast viability (supernatant assay) was noted for the titanium oxide and silica particles (data not shown). The use of a high concentration of particles is clinically relevant only for the titanium particles, as confirmed by biopsy studies. ${ }^{21,22}$

Micropipette manipulation confirmed that the osteoblasts phagocytosed the titanium particles, a phenomenon already mentioned in a study using confocal microscopy. ${ }^{20}$ The advantage of the micropipette technique was that it allowed us to observe the rapid process of phagocytosis. The osteoblasts phagocytosed the particles within $15 \mathrm{~min}$ after initial contact with particles.

The experiments performed with cytochalasin-D demonstrated that the phagocytosis of titanium particles by osteoblasts is an important factor affecting their viability. This information is consistent with the

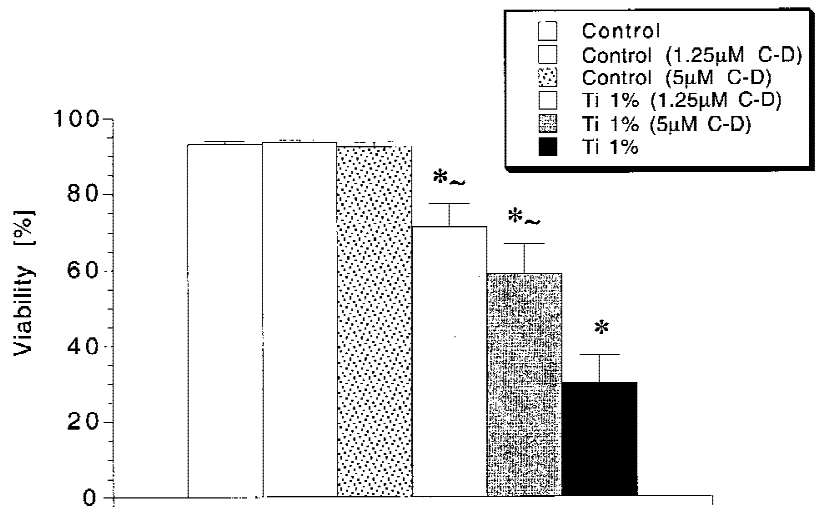

Figure 9. Mean values and standard errors of osteoblast viability cultured with conditioned medium from cocultured osteoblasts-particles treated or not treated with cytochalasin-D (*: statistical differences with respect to control, $n=16$ and $p<0.01 ; \sim$ : statistical differences with respect to $1 \%$ Ti without cytochalasin-D, $n=16$ and $p<0.001$ ). 
hypothesis of a critical number of ingested particles for osteoblasts proposed above. The phagocytic process exhibited by osteoblasts also has been suggested to be involved in the suppression of the expression of the genes that code for collagen. ${ }^{20}$

The cytochalasin-D was present during the $72 \mathrm{~h}$ of incubation for the quantification of direct effect and was used as a pretreatment for $1 \mathrm{~h}$ to quantify the indirect effect. The phagocytosis process, then, did not bring about the direct effect, but it could have been responsible for the indirect effect. This would explain why a complete viability was not recovered following the indirect effect. For the direct effect, incomplete viability recovery with cytochalasin-D suggests that mechanisms other than the phagocytosis process could influence the viability of the osteoblasts in contact with particles. Interaction with titanium ions released from the particles could be a possibility as it has been shown that plates of commercially pure titanium continuously will release ions. ${ }^{31}$ The titanium ions were shown to have moderate effects on the viability of osteogenic cells ${ }^{32}$ or monocytes/macrophages. ${ }^{33}$ It is difficult to generalize these kind of results to the present experimental situations, but it reasonably could be thought that the reaction of osteoblasts to titanium ions may be responsible for the incomplete viability recovery with the cytochalasin-D experiments. However, titanium ions seem to have more important adverse effects on the osteoblast function, such as the production of specific bone proteins ${ }^{34}$ or the favoring of a thicker fibrous interface. ${ }^{35}$

The viability of the osteoblasts in contact with titanium particles decreased rapidly in the first several hours after addition of particles and then stabilized. This rapid decrease of the viability can be correlated with the fast phagocytic process. The ratio of cell number to particle number was not constant over time in the kinetic study. After $24 \mathrm{~h}$ of incubation, the osteoblasts can proliferate, thus reducing the number of particles available per cell. Consequently the proliferation of osteoblasts alters exposure level below the threshold particle concentration value. This fact can explain the slight increase of viability after $24 \mathrm{~h}$ of culture for concentrations less than $1 \%$. For this latter concentration, the effect of particles on osteoblasts was too important to allow any recovery of the viability after $24 \mathrm{~h}$. The proliferation of osteoblasts also could explain the evolution of the number of ingested particles over time. The osteoblasts ingested most of the particles in the first $24 \mathrm{~h}$, then due to the proliferation of osteoblasts, the ratio of particles to cells decreased and so did the particle intake rate (Fig. 2).

The use of a particle-exposure-conditioned medium indicated that the titanium particles also had an indirect effect on osteoblast viability. This is the first study to report this kind of indirect effect on osteoblasts. A threshold value of particle concentration between 0.75 and $1 \%$ in the first group of osteoblasts appeared to strongly decrease the viability of osteoblasts cultured with the conditioned medium. A change in the $\mathrm{pH}$ of the medium could not be responsible for the viability decrease as its value was found to be comparable among all the different experiments (data not shown). The release of cytotoxic products by the osteoblasts co-cultured with particles, then, is a natural explanation for such a phenomenon. Other studies were interested in the quantification of proteins ${ }^{17}$ or specific cytokine production ${ }^{19}$ by the osteoblasts in contact with particles. However, these studies did not verify that the production of these agents could indirectly influence the viability of the osteoblasts as it was verified in this study.

In view of the presented results, the interaction of osteoblasts with particles could be partially responsible for peri-implant osteolysis observed in vivo. Due to the accumulation of particles in the bone over time, the number of particles could reach a critical value affecting, directly or indirectly, osteoblast viability. In this case of particle accumulation, new bone formation is affected as the osteoblast number decreases. This possible adverse effect of wear debris on net bone formation when the concentration of particles reach critically local levels also has been suggested in an in vivo study using a bone harvest chamber. ${ }^{36}$

Titanium particle exposure induced a programmed cell death (apoptosis) in the osteoblasts. This is the first study reporting this kind of behavior in connection with particle exposure. In order to confirm the occurrence of apoptosis in the osteoblasts, a DNA fragmentation assay was performed but did not exhibit a ladder pattern characteristic of apoptotic cells (data not shown). This apparent contradiction, a positive TUNEL assay, and a negative DNA fragmentation test already have been mentioned in a study interested in osteoblast apoptosis. ${ }^{26}$ The apoptosis of osteoblasts in reaction to particles can be explained by assuming that the contact with titanium particles can render dysfunctional the reception of normal extracellular or intracellular signals that promote survival of the osteoblasts. However, further studies should be performed in this field to clarify this finding.

The present study was performed on rat calvarial osteoblasts. Despite the fact that this cell line is well characterized and expresses the normal phenotypic features of osteoblasts, ${ }^{23}$ extension of the present results to human osteoblasts should be done with caution. In fact, even with human osteoblast-like cells, interaction with particles can furnish different cytotoxic results among different cells lines. ${ }^{17}$ Results of this in vitro study, then, should be considered as an indication of previously unforeseen negative osteoblast reactions to titanium wear debris particles in patients with implants made of titanium alloys.

The particles of this study were obtained from a 
commercial supplier rather than retrieved from patients. The titanium particles used in this study were slightly larger than those that have been retrieved from peri-implant tissues obtained at revision of failed prostheses. ${ }^{37,38}$ Nevertheless, it has been observed that particles induce the most acute reaction when they can be phagocytosed by the cells. ${ }^{2}$ In the present study, most of the particles were of a phagocytosable size (less than $5 \mu \mathrm{m}$ ) and thus can be considered as representative of a clinical situation.

The concentration of particles used in this study was higher than that used in previous studies. ${ }^{17,19,20}$ It was mentioned in the introduction that the retrieved tissue surrounding loose prostheses contains a high number of particles, especially for titanium alloy implants. ${ }^{21}$ Even in a well fixed prosthesis, it has been demonstrated that micromotions arise between the stem and the bone. ${ }^{39}$ These micromotions have been shown to damage the stem surface. ${ }^{40}$ The reaction of the osteoblasts to titanium particles certainly is of primary importance in the case of cementless prostheses as the particles generated will be directly in contact with the osteoblasts. A high number of particles also can originate from the titanium alloy screws used to fix the acetabular component. ${ }^{41}$ It thus is important to perform studies on osteoblast viability with concentrations of titanium particles reflecting what can be retrieved in the bone.

\section{CONCLUSIONS}

In this study it was found that titanium particles directly and indirectly affect osteoblast viability when the particle concentration reaches a threshold value. It seems important, therefore, not only to decrease the number of particles generated during the wear process, as it generally has been proposed, ${ }^{22,42,43}$ but also to avoid high concentrations of these particles in localized regions of bone tissue surrounding implants.

The authors thank Dr. Rena Bizios (Departments of Biomedical and Materials Engineering, Rensselaer Polytechnic Institute, Troy, New York) for providing the osteoblasts.

\section{References}

1. Clarke IC, Campbell P, Kossovsky N. Debris-mediated osteolysis-A cascade phenomenon involving motion, wear, particulates, macrophage induction, and bone lysis. In: Particulate debris from medical implants: mechanisms of formation and biological consequences, ASTM STP 1144. St. John KR, editor. Philadelphia: American Society for Testing and Materials; 1992. p 7-26.

2. Friedman RJ, Black J, Galante JO, Jacobs JJ, Skinner HB. Cur- rent concepts in orthopaedics biomaterials and implant fixation. J Bone Joint Surg 1993;75-A:1086-1109.

3. Maloney WJ, Smith RL, Castro F, Schurman DJ. Fibroblast response to metallic debris in vitro. J Bone Joint Surg 1993;75-A: 835-844.

4. Goodman SB, Fornasier VL. Clinical and experimental studies in the biology of aseptic loosening of joint arthroplasties and the role of polymer particles. In: St. John KR, editor. Particulate debris from medical implants: Mechanisms of formation and biological consequences, ASTM STP 1144. Philadelphia: American Society for Testing and Materials; 1992. p 27-37.

5. Huiskes R, Weinans H, Van Rietbergen B. The relationship between stress shielding and bone resorption around total hip stems and the effects of flexible materials. Clin Orthop 1992; 274:124-134.

6. Wang W, Ferguson DP, Quin JW, Simpson AH, Athanasou NA. Biomaterial particle phagocytosis by bone-resorbing osteoblast. J Bone Joint Surg 1997;79-B:849-856.

7. Glant TT, Jacobs JJ. Response of three murine macrophage populations to particulate debris: Bone resorption in organ cultures. J Orthop Res 1994;12:720-731.

8. Shanbhag AS, Macaulay W, Stefanovic-Racid M, Rubash HE. Nitric oxide release by macrophages in response to particulate wear debris. J Biomed Mater Res 1998;5:497-503.

9. Spector M, Shortkroff S, Hsu H-P, Lane N, Sledge CB, Thornhill TS. Tissue changes around loose prostheses. Clin Orthop 1990;261:140-152.

10. Lalor PA, Freeman MAR, Revell PA. Immunological studies of the bone-implant interface. Proc 16th Ann Meeting Soc Biomater; 1990. 203

11. Herman JE, Sowder WG, Anderson D, Appel AM, Hopson CN. Polymethyl-methacrylate-induced release of bone resorbing factors. J Bone Joint Surg 1991;71-A:1530-1541.

12. Lee SH, Brennan FR, Jacobs JJ, Urgan RM, Ragasa DR, Glant TT. Human monocyte/macrophage response to cobaltchromium corrosion products and titanium particles in patients with total joint replacements. J Orthop Res 1997;15:40-49.

13. Gonzales JB, Purdon MA, Horowitz SM. In vitro studies on the role of titanium in aseptic loosening. Clin Orthop 1996;330:244250.

14. Horowitz SM, Gonzales JB. Inflammatory response to implant particulates in a macrophage/osteoblast coculture model. Calcif Tissue Int 1996;59:392-396.

15. Goodman SB, Aspenberg P, Song Y, Doshi A, Regula D, Lidgren L. Tissue differentiation in the presence of different concentrations of phagocytosable polyethylene and titanium alloy particles. Proc 2nd Combined Orthop Res Soc; 1995. p 218.

16. Goodman SB, Davidson JA, Song Y, Martial N, Fornasier VL. Histomorphological reaction of bone to different concentrations of phagocytosable particles of high-density polyethylene and Ti-6Al-4V alloy in vivo. Biomaterials 1996;17:1943-1947.

17. Allen MJ, Myer BJ, Millett PJ, Rushton N. The effects of particulate cobalt, chromium and cobalt-chromium alloy on human osteoblast-like cells in vitro. J Bone Joint Surg 1997;79-B: 475-482.

18. Martinez ME, Medina S, del Campo MT, Garcia JA, Rodrigo A, Munuera L. Effect of polyethylene particles on human osteoblastic cell growth. Biomaterials 1998;19:183-187.

19. Wang JY, Wicklund BH, Gustilo RB, Tsukayama DT. Prosthetic metals interfere with the functions of human osteoblast cells in vitro. Clin Orthop 1997;339:216-226.

20. Yao J, Cs-Szabo G, Jacobs JJ, Kuettner KE, Glant TT. Suppression of osteoblast function by titanium particles. J Bone Joint Surg 1997;79-A:107-112.

21. Brien WW, Salvati EA, Betts F, Bullough P, Wright T, Rimnac C, Bully R, Garvin K. Metal levels in cemented total hip arthroplasty. Clin Orthop 1992;276:66-74. 
22. Salvati EA, Foster B, Doty SB. Particulate metallic debris in cemented total hip arthroplasty. Clin Orthop 1993;293:160-173.

23. Puleo DA, Holleran LA, Domerus RH, Bizios R. Osteoblast response to orthopaedic implants in vitro. J Biomed Mater Res 1991;25:711.

24. Jones KH, Senft JA. An improved method to determine cell viability by simultaneous staining with fluorescein diacetatepropidium iodide. J Histochem Cytochem 1985;33:77-79.

25. Darzynkiewicz Z, Li X, Gong J. Assays of cell viability: Discrimination of cells dying by apoptosis. Meth Cell Biol 1994; 41:15-38.

26. Hill PA, Tumber A, Meikle MC. Multiple extracellular signals promote osteoblast survival and apoptosis. Endocrinology 1997;138:3849-3958.

27. Sung K-LP, Sung LA, Crimmins M, Burakoff SJ, Chien S. Determination of junction avidity of cytolytic T-cell and target cell. Science 1986;234:1405-1408.

28. Rest RF, Speert DP. Measurement of nonopsonic phagocytic killing by human and mouse phagocytes. In: Clark VL, Bavoil PM, editors. Methods in enzymology. San Diego: Academic Press; 1994. p 91-108.

29. Choi SC. Introductory applied statistics in science. Englewood Cliffs, New Jersey: Prentice-Hall; 1978. 278 p.

30. Kobayashi A, Freeman MAR, Bonfield W, Kadoya Y, Yamac T, Al-Saffar N, Scott G, Revell PA. Number of polyethylene particles and osteolysis in total joint replacements. J Bone Joint Surg 1997;79-B:844-848.

31. Fartash B, Liao H, Li J, Fouda N, Hermansson L. Long-term evaluation of titania-based ceramics compared with commercially pure titanium in vivo. J Mater Sci Mater Med 1995;6:451454.

32. Puelo DA, Huh WW. Acute toxicity of metal ions in cultures of osteogenic cells derived from bone marrow stromal cells. J Appl Biomater 1995;6:109-116.

33. Wang JY, Wicklund BH, Gustilo RB, Tsukayama DT. Titanium, chromium and cobalt ions modulate the release of boneassociated cytokines by human monocytes/macrophages in vitro. Biomaterials 1996;17:2233-2240.

34. Sun ZL, Wataha JC, Hanks CT. Effects of metal ions on osteoblast-like cell metabolism and differentation. J Biomed Mater Res 1997;34:29-37.
35. Schepers E, Clercq M, Ducheyne P. Tissue response to a bioactive glass-metal interface. In: De Putter C, editor Implant materials in biofunction. Amsterdam: Elsevier Science Publishers; 1987. p 79-85.

36. Goodman S, Aspenberg P, Song Y, Regula D, Lidgren L. Polyethylene and titanium alloy particles reduce bone formation. Dose-dependence in bone harvest chamber experiments in rabbits. Acta Orthop Scand 1996;67:599-605.

37. Lee JM, Salvati EA, Betts F, DiCarlo EF, Doty SB, Bullough PG. Size of metallic and polyethylene debris particles in failed cemented total hip replacements. J Bone Joint Surg 1992;74-B: 380-384.

38. Maloney WJ, Smith RL, Schmalzried TP, Chiba J, Huene D, Rubash H. Isolation and characterization of wear particles generated in patients who have had failure of a hip arthroplasty without cement. J Bone Joint Surg 1995;77-A:1301-1310.

39. Ramaniraka NA, Leyvraz PF, Rakotomanana LR, Rubin PJ, Zysset PK. Micromotion at the bone-stem interface during the gait cycle after cementless total hip replacement: Influence of stem design and loading level. Hip International 1996;6:51-58.

40. Charriere E, Rakotomanana LR, Rubin PJ, Leyvraz P-F. Cemented femoral stem after THR: Influence of stress and micromotions on the wear rate and on the deterioration of the stem surface topography. Proc. of 8th EORS; 1988. p O73.

41. Lalor PA, Revel PA, Gray AB, Wright S, Railton GT, Freeman MA. Sensitivity to titanium. A cause of implant failure? J Bone Joint Surg 1991;73-B:25-28.

42. Bordji K, Jouzeau JY, Mainard D, Payan E, Netter P, Rie KT, Stucky T, Hage-Ali M. Cytocompatibility of Ti-6A1-4V and Ti-5A1-2.5 Fe alloys according to three surface treatments, using human fibroblasts and osteoblasts. Biomaterials 1996;17: 929-940.

43. Campbell P, Nasser S, Kossovsky N, Amstutz HC. Histopathological effects of ultrahigh-molecular-weight polyethylene and metal wear debris in porous and cemented surface replacements. In: St. John KR, editor. Particulate debris from medical implants: Mechanisms of formation and biological consequences, ASTM STP 1144. Philadelphia: American Society for Testing and Materials; 1992. p 38-51. 